\title{
Ancient eruptions of $\eta$ Carinae: a tale written in proper motions
}

\author{
Megan M. Kiminki, ${ }^{1 \star}$ Megan Reiter ${ }^{2}$ and Nathan Smith $^{1}$ \\ ${ }^{1}$ Steward Observatory, University of Arizona, 933 N. Cherry Avenue, Tucson, AZ 85721, USA \\ ${ }^{2}$ Department of Astronomy, University of Michigan, 311 West Hall, 1085 S. University Avenue, Ann Arbor, MI 48109, USA
}

Accepted 2016 August 9. Received 2016 August 8; in original form 2016 June 6

\begin{abstract}
We analyse eight epochs of Hubble Space Telescope $\mathrm{H} \alpha+\left[\mathrm{N}_{\text {III }}\right.$ imaging of $\eta$ Carinae's outer ejecta. Proper motions of nearly 800 knots reveal that the detected ejecta are divided into three apparent age groups, dating to around 1250 A.D., to around 1550 A.D., and to during or shortly before the Great Eruption of the 1840s. Ejecta from these groups reside in different locations and provide a firm constraint that $\eta$ Car experienced multiple major eruptions prior to the nineteenth century. The 1250 and 1550 events did not share the same axisymmetry as the Homunculus; the 1250 event was particularly asymmetric, even one-sided. In addition, the ejecta in the $\mathrm{S}$ ridge, which have been associated with the Great Eruption, appear to predate the ejection of the Homunculus by several decades. We detect essentially ballistic expansion across multiple epochs. We find no evidence for large-scale deceleration of the observed knots that could power the soft X-ray shell by ploughing into surrounding material, suggesting that the observed X-rays arise instead from fast, rarefied ejecta from the 1840s overtaking the older dense knots. Early deceleration and subsequent coasting cannot explain the origin of the older outer ejecta - significant episodic mass loss prior to the nineteenth century is required. The time-scale and geometry of the past eruptions provide important constraints for any theoretical physical mechanisms driving $\eta$ Car's behaviour. Non-repeating mechanisms such as the merger of a close binary in a triple system would require additional complexities to explain the observations.
\end{abstract}

Key words: circumstellar matter-stars: individual: $\eta$ Carinae-stars: mass-loss.

\section{INTRODUCTION}

One of the most remarkable stars in our Galaxy, $\eta$ Carinae has been puzzling astronomers for over 150 years. In the mid-nineteenth century, it became increasingly variable, then peaked temporarily as the second brightest star in the sky (Innes 1903; Davidson \& Humphreys 1997; Frew 2004; Smith \& Frew 2011) before slowly fading over more than a decade. During this Great Eruption, $\eta$ Car ejected an estimated 10-15 $\mathrm{M}_{\odot}$ into the well-known bipolar Homunculus nebula (Smith et al. 2003). A second, Lesser Eruption followed in 1890 (Innes 1903; Humphreys, Davidson \& Smith 1999; Frew 2004), but only ejected $\sim 0.1 \mathrm{M}_{\odot}$ (Ishibashi et al. 2003; Smith 2005).

$\eta$ Car belongs to a class of stars known as luminous blue variables (LBVs; Humphreys \& Davidson 1994), very massive, unstable, post-main-sequence stars characterized by luminous mass-loss events. Even among LBVs, $\eta$ Car is unusual and its parameters are extreme. In its current quiescent state, $\eta$ Car is substantially more luminous than most other known LBVs (e.g. van Genderen 2001;
Smith \& Tombleson 2015). It is one of only two Galactic LBVs that has been observed in a giant eruption. The other is P Cygni, whose largest eruption involved significantly less energy and mass loss, similar to $\eta$ Car's Lesser Eruption (Smith \& Hartigan 2006). Moreover, $\eta$ Car has a massive binary companion in an eccentric 5.5-year orbit (Damineli 1996; Damineli, Conti \& Lopes 1997; Damineli et al. 2000; Corcoran et al. 2001; Whitelock et al. 2004), and is located in the rich cluster Trumpler 16, home to dozens of O-type stars (Smith 2006a). In contrast, most LBVs are relatively isolated and lack O-type neighbours (Smith \& Tombleson 2015).

The mechanism of $\eta$ Car's Great Eruption - which released roughly $10^{50}$ erg of kinetic energy (Smith et al. 2003; Smith 2008) remains a mystery. Many theories treat it as part of single-star evolution, invoking super-Eddington radiation-driven winds (Davidson 1971; Maeder 1983; de Jager 1984; Lamers \& Fitzpatrick 1988; Stothers \& Chin 1993; Glatzel \& Kiriakidis 1993; Glatzel 1994; Humphreys \& Davidson 1994; Shaviv 2000; Owocki, Gayley \& Shaviv 2004). However, the source of the increased bolometric luminosity in these scenarios is unclear. Alternatively, the eccentric orbit of $\eta$ Car's companion has been taken to imply that the Great Eruption was influenced by periastron interactions between the two binary components. Based on nineteenth-century observers' 


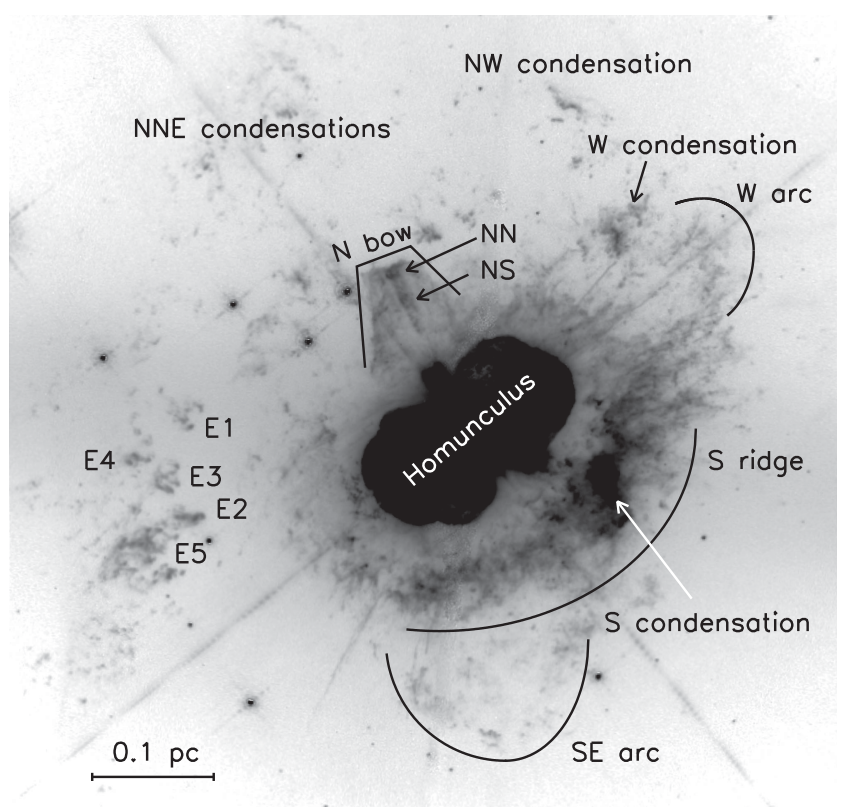

Figure 1. HST WFPC2 image of $\eta$ Car in the F658N filter, which captures intrinsic and scattered [N II] $\lambda 6584$ emission along with redshifted $\mathrm{H} \alpha$ emission (Morse 1999; Morse et al. 2001). Prominent features are labelled according to the convention of Walborn (1976) and Weis (2012).

estimates of the primary star's colour and brightness, its radius must have been much larger than at present, large enough that its companion would significantly interact or even physically collide (Iben 1999; Smith 2011). A speculative idea is that the collision mixed fresh nuclear fuel to greater depths, causing a sudden burst of increased nuclear burning (Smith 2011). It has also been proposed that periastron tidal interactions spun up the primary to unstable rates, leading to a burst of mass loss (Cassinelli 1999), or that the Great Eruption was fuelled by accretion from the primary on to its companion (Soker 2007; Kashi \& Soker 2010). Still other theories postulate a hierarchical triple system, in which the close inner pair either merged (Gallagher 1989; Iben 1999; Morris \& Podsiadlowski 2009; Podsiadlowski 2010; Portegies Zwart \& van den Heuvel 2016) or underwent a dynamical exchange with the outer companion (Livio \& Pringle 1998).

Models of the driving cause of the Great Eruption must also incorporate the outer ejecta, a collection of irregular condensations found out to nearly half a parsec outside the Homunculus (Thackeray 1950; Walborn 1976; Meaburn et al. 1996; Smith \& Morse 2004; Weis 2012). These outer ejecta (Fig. 1) are highly nitrogen-rich, suggesting a substantial degree of CNO processing (Davidson et al. 1986; Smith \& Morse 2004). They contain a minimum mass of 2-4 $\mathrm{M}_{\odot}$ (Weis 2012), with dust observations suggesting a much larger total mass (Gomez et al. 2010). The various models for the Great Eruption produce different explanations for the outer ejecta. The merger model of Portegies Zwart \& van den Heuvel (2016), for instance, predicts that the outer ejecta were formed after the formation of the Homunculus.

The proper motions of the outer ejecta provide concrete constraints on $\eta$ Car's mass-loss history. The bright $\mathrm{S}$ condensation and the 'jet'-shaped $\mathrm{N}$ bow (see Fig. 1) have motions consistent with having been ejected during the Great Eruption (Walborn, Blanco \& Thackeray 1978; Ebbets et al. 1993; Currie et al. 1996; Morse et al. 2001). Some results have suggested, however, that the extended $S$ ridge is up to 100 years older (Walborn et al. 1978; Morse et al.
2001). The age of the E condensations is even less clear: Walborn et al. (1978) found transverse velocities of $300-400 \mathrm{~km} \mathrm{~s}^{-1}$, indicating ejection dates in the mid-1400s, but Walborn \& Blanco (1988) determined 10 years later that the same features had slowed dramatically, suggesting they were from the Great Eruption after all. While the motions of the outer condensations have hinted at prior mass-loss events, a single ejection date around the time of the Great Eruption could not be ruled out.

In this paper, we measure the proper motions of $\eta$ Car's outer ejecta to unprecedented accuracy, using 16 different baselines over 21 years of Hubble Space Telescope (HST) data. The depth and resolution of the HST images allow us to re-evaluate the origins of the N, E, and S features, and, for the first time, measure motions of the fainter NNE, NW, and SE condensations. We find no evidence of widespread deceleration, and show that while some of the outer ejecta come from the Great Eruption (or the lead-up to it), many features require at least one prior mass-loss event centuries earlier. Our data, image registration, and approach to measuring proper motions are described in Section 2; the results are presented in Section 3. We discuss the implications of our results on models of $\eta$ Car in Section 4 and conclude with a summary in Section 5.

\section{OBSERVATIONS AND ANALYSIS}

\subsection{HST ACS}

We obtained new $\mathrm{H} \alpha+[\mathrm{N}$ II] images of $\eta$ Car and the surrounding $\mathrm{Tr}$ 16 cluster on 2014 Aug 4, using the Wide Field Channel (WFC) of the Advanced Camera for Surveys (ACS) on HST (programme ID 13390; see Table 1). These observations were designed to replicate our observations of 2005 Jul 18 (Smith, Bally \& Walborn 2010) as closely as possible, in pointing and position angle, in order to minimize position-dependent uncertainties when measuring proper motions. The Homunculus and central star are heavily saturated in these exposures, which were each 500-520 s long. In both epochs, observations were made as a series of $205 \times 400 \operatorname{arcsec}^{2}$ 'footprints', each consisting of three pairs of CR-SPLIT dithers in a linear offset pattern designed to fill in the ACS chip gaps. $\eta$ Car and its outer ejecta were observed in a single footprint; the rest make up a mosiac of $\operatorname{Tr} 16$ and neighbouring $\operatorname{Tr} 14$ (see Smith et al. 2010). All data were processed by the standard HST ACS pipeline, which does bias subtraction and flat-fielding and corrects for charge transfer efficiency (CTE) to produce an image with the designation ' $\mathrm{flc}$ '. The pipeline also produces a 'drc' image that has been additionally corrected for geometric distortion and dither-combined via AstroDrizzle.

We transformed both epochs of ACS data to a common distortionfree reference frame via a modified version of the method used in Anderson et al. (2008a,b), Anderson \& van der Marel (2010), and Sohn, Anderson \& van der Marel (2012, see also Reiter et al. $2015 a, b)$. An initial reference frame, aligned with the $y$-axis pointing north and with a pixel scale of 50 mas pixel ${ }^{-1}$, was constructed using the astrometric solutions in the headers of the drc images to match stars that appear in overlapping images. However, the resampling performed by AstroDrizzle makes the drc images unsuitable for direct high-accuracy positional measurements. Instead, we performed point spread function (PSF) fitting on the undrizzled $\mathrm{flc}$ images using the program IMG2XYM_WFC.09X10 (Anderson \& King 2006), which uses a library of spatially dependent effective PSFs. We then applied the geometric distortion corrections of Anderson (2006) to the measured stellar positions. 
Table 1. HST data log.

\begin{tabular}{lccccc}
\hline Instrument & Camera & Date & Filter & Exp. time (s) & Programme ID \\
\hline WFPC2 & WF3 & 1993 Dec 31 & F658N & $2 \times 200$ & 5188 \\
WFPC2 & WF3 & 1997 Jul 11 & F658N & $2 \times 200$ & 7253 \\
WFPC2 & WF3 & 1999 Jun 12 & F658N & $2 \times 200$ & 8178 \\
WFPC2 & WF3 & 2001 Jun 4 & F658N & $2 \times 200$ & 9226 \\
WFPC2 & WF3 & 2003 Aug 8 & F658N & $2 \times 100$ & 9775 \\
WFPC2 & WF3 & 2008 Sept 6 & F658N & $2 \times 100$ & 11500 \\
ACS & WFC & 2005 Jul 18 & F658N & $2 \times 500$ & 10241 \\
ACS & WFC & 2014 Aug 4 & F658N & $2 \times 520$ & 13390 \\
\hline
\end{tabular}

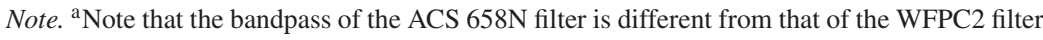
of the same name (see discussion in Section 2.2).
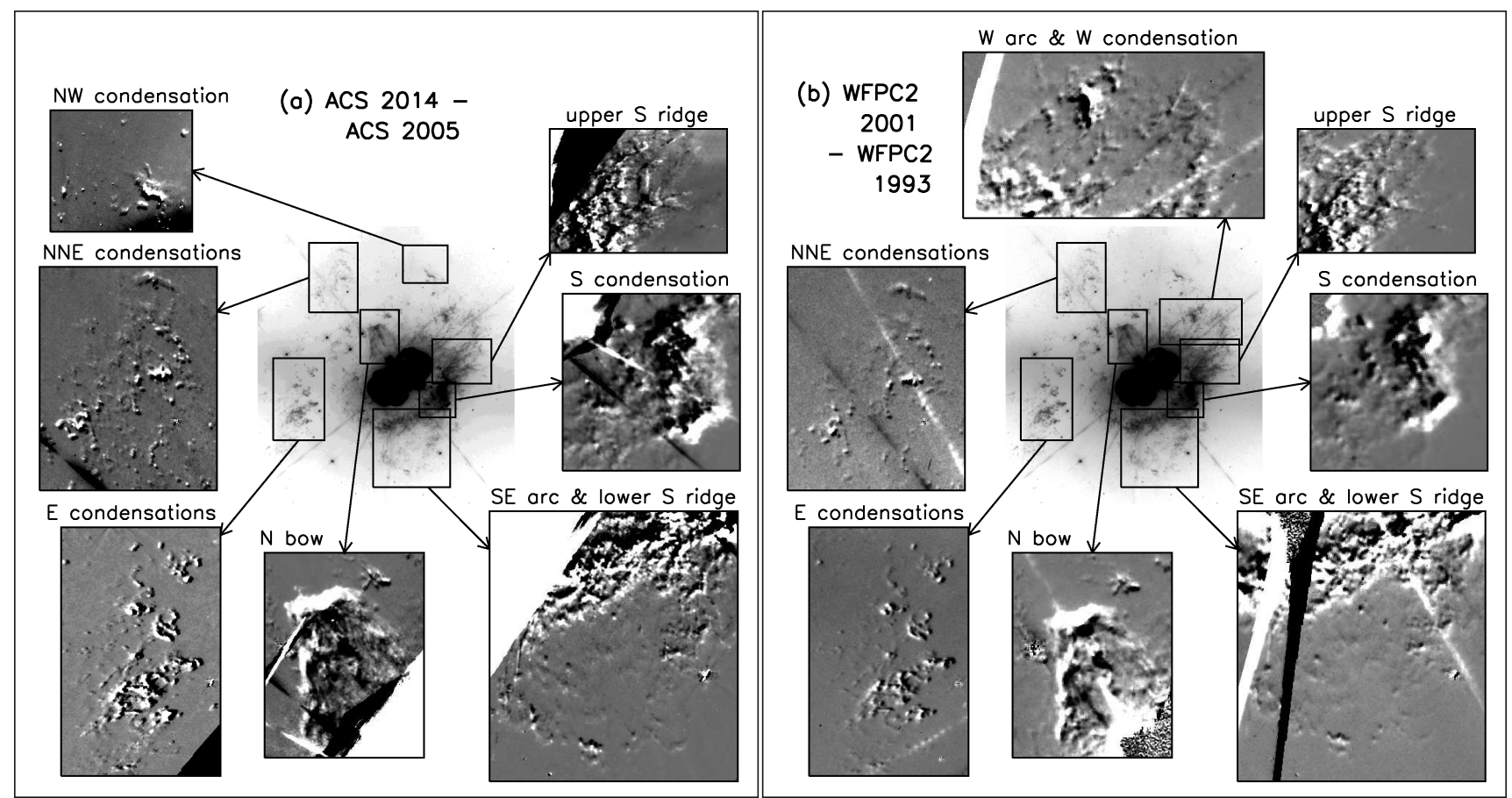

Figure 2. (a) Difference images showing the outward motion of $\eta$ Car's ejecta over the 9-year baseline between ACS images. Dark patches are where the various condensations were in 2005; bright patches are places into which that material had moved by 2014. The central image is the same one shown in Fig. 1 and is included to orient the reader. (b) Same as (a) but for the 7-yr baseline between the WFPC2 1993 and 2001 images. The W and NW condensations are not shown in the ACS (left) and WFPC2 (right) panels, respectively, because they are contaminated by bleeding from the saturated Homunculus in the relevant epochs. Animated GIFs showing the motion of each feature across all eight epochs are available in the online supplementary material.

Next, we iteratively mapped the stellar positions from the $\mathrm{flc}$ images to the reference frame by:

(i) identifying the high signal-to-noise stars (typically several hundred per image) in common between each $f l c$ image and the reference frame;

(ii) computing the six-dimensional linear transformation from the distortion-corrected positions of the $\mathrm{flc}$ images to the reference frame; and

(iii) replacing each existing reference-frame stellar position with the average of that star's transformed, distortion-corrected $f l c$ positions.

After three iterations, the internal accuracy of the reference frame was $<0.02$ pixels ( 1 mas, or $1.2 \mathrm{~km} \mathrm{~s}^{-1}$ over this 9 -yr baseline at the distance of $\eta$ Car) across a single footprint.

Using the final linear transformations determined by this process, we resampled the $\mathrm{flc}$ images from each epoch into the reference frame using the algorithm described in Anderson et al. (2008a), scaling each image to a total exposure time of $500 \mathrm{~s}$. The end result was a single stacked image at each epoch for each observed footprint. With both epochs of data on the same reference frame, the positions of ejecta features in the stacked images could be directly compared. Fig. 2 presents difference images (stacked image from 2014 minus stacked image from 2005) for named regions of interest in $\eta$ Car's outer ejecta. In these images, material has moved from the dark patches into the corresponding bright areas. It is immediately qualitatively apparent that some features have greater transverse velocities than others; the $\mathrm{N}$ bow, for instance, is rapidly overtaking the small feature to its immediate north-west. Notably, there are no pronounced changes in condensation morphology or brightness from 2005 to 2014.

The proper motions of well-defined knots in the ejecta around $\eta$ Car were measured in the stacked images. 'Well-defined' features are those that are (1) not contaminated by or confused with the 


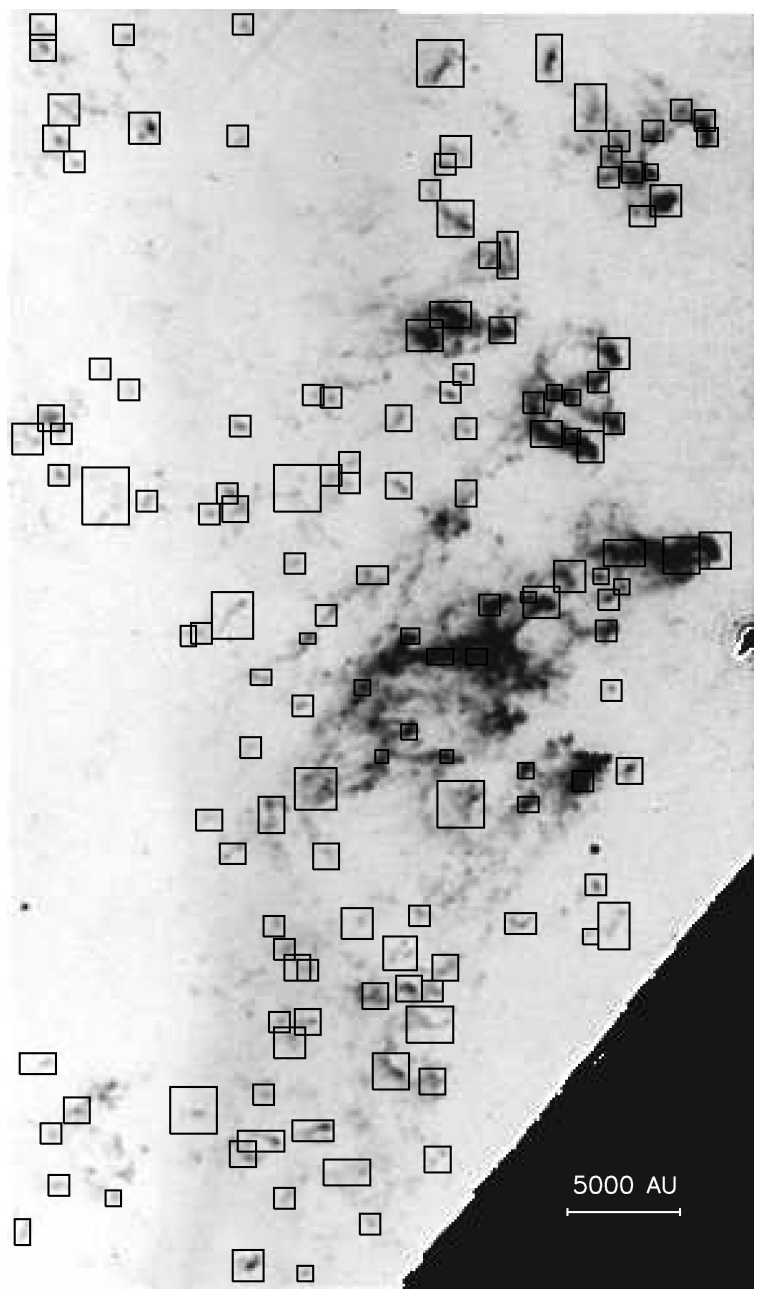

Figure 3. Close-up of the region around the E condensations in the 2005 ACS F658N image, showing the boxes used to measure proper motions via modified cross-correlation. The saturated strip in the lower right is bleeding from the saturated Homunculus.

saturated Homunculus or diffraction spikes in either epoch and (2) sufficiently isolated for a defined knot or feature to be identified. A total of 620 such features were identified in the ACS images. To measure the proper motion of each, we subtracted a median-filtered image (filtered using a kernal size of 12 pixels) in order to suppress the local diffuse $\mathrm{H} \alpha$ background. We then extracted the knot using a box size optimized for that feature; Fig. 3 illustrates the boxes used for the features in and around the E condensations. We used our implementation of the modified cross-correlation technique developed by Currie et al. (1996), Hartigan et al. (2001), and Morse et al. (2001, see also Reiter \& Smith 2014; Reiter et al. 2015a,b) to determine the difference in position between the two epochs. In brief, the box containing the feature of interest was compared to the second image and an array was generated containing the total of the square of the difference between two images for each shift. The minimum of this array corresponds to the shift that best matches the two images. Angular displacements were converted to $\mathrm{km} \mathrm{s}^{-1}$ assuming a distance of $2.3 \mathrm{kpc}$ (Smith 2006b). To estimate the uncertainty on the offset, we repeated the procedure using a variety of box sizes. The median proper motion uncertainty for features measured in the ACS images is $2.7 \mathrm{~km} \mathrm{~s}^{-1}$.

\subsection{HST WFPC2}

To supplement our proper motion measurements from the 2005 2014 ACS baseline, we searched the HST archive for additional deep images of $\eta$ Car taken with an F658N filter. Six epochs of observations from the Wide-Field Planetary Camera 2 (WFPC2) met our requirements: the images had to be deep enough for the outer ejecta to be visible, and $\eta$ Car had to be centred on one of the Wide Field chips (the field of view of the Planetary Camera chip is too small). The dates and programme IDs for these data are given in Table 1. We retrieved the longest exposures available at each epoch: a pair of 200-s exposures from each of 1993, 1997, 1999, and 2001, and a pair of 100-s exposures from each of 2003 and 2008. $\eta$ Car itself and much of the Homunculus are saturated in these images, although the bleeding is much less extensive than in the ACS images, which have both longer exposure times and a wider filter bandpass (see below). All of the data had been reprocessed by the final version of the HST WFPC2 pipeline, which produces images designated ' $\mathrm{C} 0 \mathrm{~m}$ ' that have been bias-subtracted, dark-corrected, and flat-fielded.

Note that there are important differences between the WFPC2 F658N filter and the ACS filter of the same name. The former covers a narrow wavelength range (FWHM $38 \AA$ ) roughly centred on $\left[\mathrm{N}_{\mathrm{II}}\right] \lambda 6584$, capturing emission from that line with Doppler shifts from approximately -615 to $+1130 \mathrm{~km} \mathrm{~s}^{-1}$. The latter is broader (FWHM $72 \AA$ ), capturing [N II] emission with radial velocities up to $\pm 1600 \mathrm{~km} \mathrm{~s}^{-1}$ as well as significant $\mathrm{H} \alpha$ emission. Although most of the same ejecta features seen in the ACS images are identifiable in the WFPC2 images, there is no way to guarantee that the knot shapes are unaffected by the differences in filter bandpass. Consequently, we do not measure proper motions directly between WFPC2 and ACS images. We use only WFPC2-WFPC2 and ACS-ACS baselines. In addition, the fastest known ejecta are Doppler-shifted out of one or both filters and are not detected. The WFPC2 F658N filter would not, for instance, pick up the $-875 \mathrm{~km} \mathrm{~s}^{-1}$ blueshifted emission just outside the southeast lobe of the Homunculus (Currie, Dorland \& Kaufer 2002), even if the Homunculus were not saturated in our images. Both filters miss the $-3200 \mathrm{~km} \mathrm{~s}^{-1}$ blueshifted ejecta detected further out by Smith (2008). Our images thus exclude the fastest-moving material from the Great Eruption and therefore do not exclude the possibility of additional recent mass-loss episodes. We note, however, that since few of the ejecta seen in the ACS images drop out in the WFPC2 images (and those that do are relatively faint and not detected in the shortest exposures), few to none of the dense knots measured in our images have radial velocities of $\pm 1000-1500 \mathrm{~km} \mathrm{~s}^{-1}$.

We took the same approach to aligning and stacking the WFPC2 data as we did with the ACS images. We used a modified version of the IMG2XYM program (Anderson \& King 2006) to measure stellar positions in the $\mathrm{C} 0 \mathrm{~m}$ images, employing a library of spatially dependent effective WFPC2 PSFs from Anderson \& King (2000). The measured positions were then corrected for the 34th-row anomaly (Anderson \& King 1999) and for geometric distortion (Anderson \& King 2000). We identified 6-10 isolated, low-proper-motion stars in common between each WFPC2 image and the ACS-based reference frame, and used those stars to derive a six-parameter linear transformation between each distortion-corrected WFPC2 frame and the reference frame. Unlike with the ACS data, we did not iterate on the process: we took the ACS-based reference frame as final. The internal accuracy of the alignment and stacking procedure is $<0.06$ reference-frame pixels ( 3 mas) for the first four epochs. The shorter, lower signal-to-noise ratio 2003 and 2008 exposures had 
Table 2. Baselines used for proper motion measurements.

\begin{tabular}{lcrcr}
\hline Baseline & Instrument & \multicolumn{1}{c}{$\begin{array}{c}\Delta \mathrm{t} \\
(\mathrm{yr})\end{array}$} & No. of features & $\begin{array}{r}\text { Median } \sigma \\
\left(\mathrm{km} \mathrm{s}^{-1}\right)\end{array}$ \\
\hline $1993-1997$ & WFPC2 & 3.524068 & 694 & 8.5 \\
$1993-1999$ & WFPC2 & 5.443602 & 704 & 7.0 \\
$1993-2001$ & WFPC2 & 7.422813 & 712 & 5.4 \\
$1993-2003$ & WFPC2 & 9.599420 & 714 & 4.8 \\
$1993-2008$ & WFPC2 & 14.680967 & 698 & 3.9 \\
$1997-1999$ & WFPC2 & 1.919534 & 703 & 14.3 \\
$1997-2001$ & WFPC2 & 3.898745 & 742 & 7.7 \\
$1997-2003$ & WFPC2 & 6.075352 & 713 & 6.3 \\
$1997-2008$ & WFPC2 & 11.156898 & 686 & 4.6 \\
$1999-2001$ & WFPC2 & 1.979211 & 716 & 12.1 \\
$1999-2003$ & WFPC2 & 4.155818 & 684 & 8.0 \\
$1999-2008$ & WFPC2 & 9.237365 & 679 & 4.6 \\
$2001-2003$ & WFPC2 & 2.176607 & 633 & 13.0 \\
$2001-2008$ & WFPC2 & 7.258153 & 666 & 5.5 \\
$2003-2008$ & WFPC2 & 5.081546 & 648 & 7.6 \\
$2005-2014$ & ACS & 9.046256 & 620 & 2.7 \\
\hline
\end{tabular}

greater positional uncertainties of up to 0.2 reference frame pixels (10 mas).

Using the calculated transformations, the WFPC $2 \mathrm{c} 0 \mathrm{~m}$ images were resampled and stacked into a single image at each epoch, aligned with the ACS-based reference frame. The c0m images, which have a plate scale of 99.6 mas pixel ${ }^{-1}$, were oversampled during stacking so that the output image matched the 50-mas pixels of the ACS images. An example of the results is shown in the right panel of Fig. 2, which shows difference images for the major named regions of $\eta$ Car's outer ejecta for the 1993-2001 baseline. Although the resolution is somewhat lower compared to the ACS images, much detail is still apparent. Animated GIFs of each region, made from all eight epochs of aligned data, are available in the online supplementary material.

The proper motions of the features identified in the ACS images were measured in the WFPC2 images, using the modified crosscorrelation technique described in Section 2.1, for every possible baseline among the six epochs (a total of 15 baselines). Some features were contaminated by diffraction spikes or saturation bleeds in some epochs; proper motions for these features were measured using only the epochs in which they were not contaminated. The baselines, number of features measured, and median proper motion uncertainties for each baseline are given in Table 2. The median proper motion uncertainties range from 3.9 to $14.3 \mathrm{~km} \mathrm{~s}^{-1}$, and the magnitude of the uncertainty is highly negatively correlated with the length of the baseline (i.e. shorter baselines produce greater uncertainties). In the areas blocked by saturation bleeding in the ACS images, we used the 1993-2001 baseline (the longest baseline with 200-s exposures) to identify additional features which were then measured across the other WFPC2 baselines where possible. A small number of features in a gap that was saturated in 2001 are identified in the 1993-2003 baseline instead, then measured across other baselines where possible. In total, we measure the proper motions of 792 individual features in the ejecta of $\eta$ Car. Each feature is measured in 1-16 baselines (including the 2005-2014 ACS baseline); on average, a feature is measured in 14 baselines.

\subsection{Position of the central source}

Owing to the heavy saturation of the Homunculus, we were unable to measure the position of $\eta$ Car itself in any of our stacked images.
Instead, we measure the centroid of the central star in an F631N WFPC2 Planetary Camera image from Morse et al. (1998). The centroid positions of five surrounding stars in this image were used to derive a linear transformation to our reference frame. We estimate that this transformation is accurate to $\sim 0.2$ reference-frame pixels. Added in quadrature with the \pm 0.5 -pixel uncertainties in absolute knot positions, this means that the overall uncertainty in a knot's distance from $\eta$ Car is $\sim 0.55$ pixels ( 27 mas, or 60 au at the distance of $\eta$ Car). For a knot moving at $300 \mathrm{~km} \mathrm{~s}^{-1}$, this translates to an uncertainty in its age of \pm 0.9 years.

\section{RESULTS}

\subsection{HST proper motions over two decades}

Fig. 4 presents our results in aggregate: a histogram of the weighted mean proper motions of all measured features, along with proper motion histograms for the ejecta in each of the regions highlighted in Fig. 2. The transverse velocities of $\eta$ Car's outer ejecta range from $219 \mathrm{~km} \mathrm{~s}^{-1}$ to $1462 \mathrm{~km} \mathrm{~s}^{-1}$, with a broad peak at $300-600 \mathrm{~km} \mathrm{~s}^{-1}$ and a secondary peak at $750-900 \mathrm{~km} \mathrm{~s}^{-1}$. The highest proper motions are found in the $\mathrm{N}$ bow, the $\mathrm{S}$ ridge, and the parts of the $\mathrm{W}$ arc that overlap with the upper $\mathrm{S}$ ridge. The presence and location of speeds upward of $1000 \mathrm{~km} \mathrm{~s}^{-1}$ agrees with past observations of the S ridge and $\mathrm{N}$ bow (Walborn et al. 1978; Walborn \& Blanco 1988; Ebbets et al. 1993; Morse et al. 2001). The mix of speeds seen in the lower $\mathrm{S}$ ridge is also consistent with the results of Morse et al. (2001).

Our results for the E condensations (no high-proper-motion features; mean proper motion $\sim 400 \mathrm{~km} \mathrm{~s}^{-1}$ ) agree solidly with the results of Walborn et al. (1978), who used photographic plate images from 1950 to 1975 . However, the observed motion in our HST images disagrees strongly with the deceleration hypothesis of Walborn \& Blanco (1988), who added data from 1985 and found that the E condensations had dramatically slowed over 10 years. The Walborn \& Blanco (1988) deceleration hypothesis predicts that the E features would have reached zero velocity by the end of the twentieth century, a hypothesis that our data definitively exclude.

With a total of 16 different baselines from two instruments, we can approach the question of acceleration/deceleration with unprecedented detail. Fig. 5 plots the measured proper motions over time for three features representative of the E condensations and of the ejecta overall. The weighted mean transverse velocity of each feature is overplotted, along with a least-squares fit to the data. Although there is some variation among the proper motions, there are no overall trends with time. In a $\chi^{2}$ test, the measured motions for all three features shown in Fig. 5 are consistent with having been drawn from a distribution with constant transverse velocity.

This level of variance and (lack of) acceleration is found across all our data. Out of the 792 features for which proper motions were measured, only 50 (6 per cent) have data that are inconsistent at the $p<0.05$ level with being drawn from a constant velocity distribution. These 50 features occupy no special region of physical space or velocity space. The slopes of the least-squares fits to velocity versus time for these 50 features also fall inside the range of slopes found for the rest of the ejecta. If material were being significantly accelerated (from being hit by younger, faster material) or decelerated (from running into older material), we would expect to see some kind of spatial correlation of those changes in velocity, yet we do not. Thus, while we cannot rule out small amounts of acceleration or deceleration for individual features, the outer ejecta appear to be, on the whole, expanding ballistically. We therefore treat all 


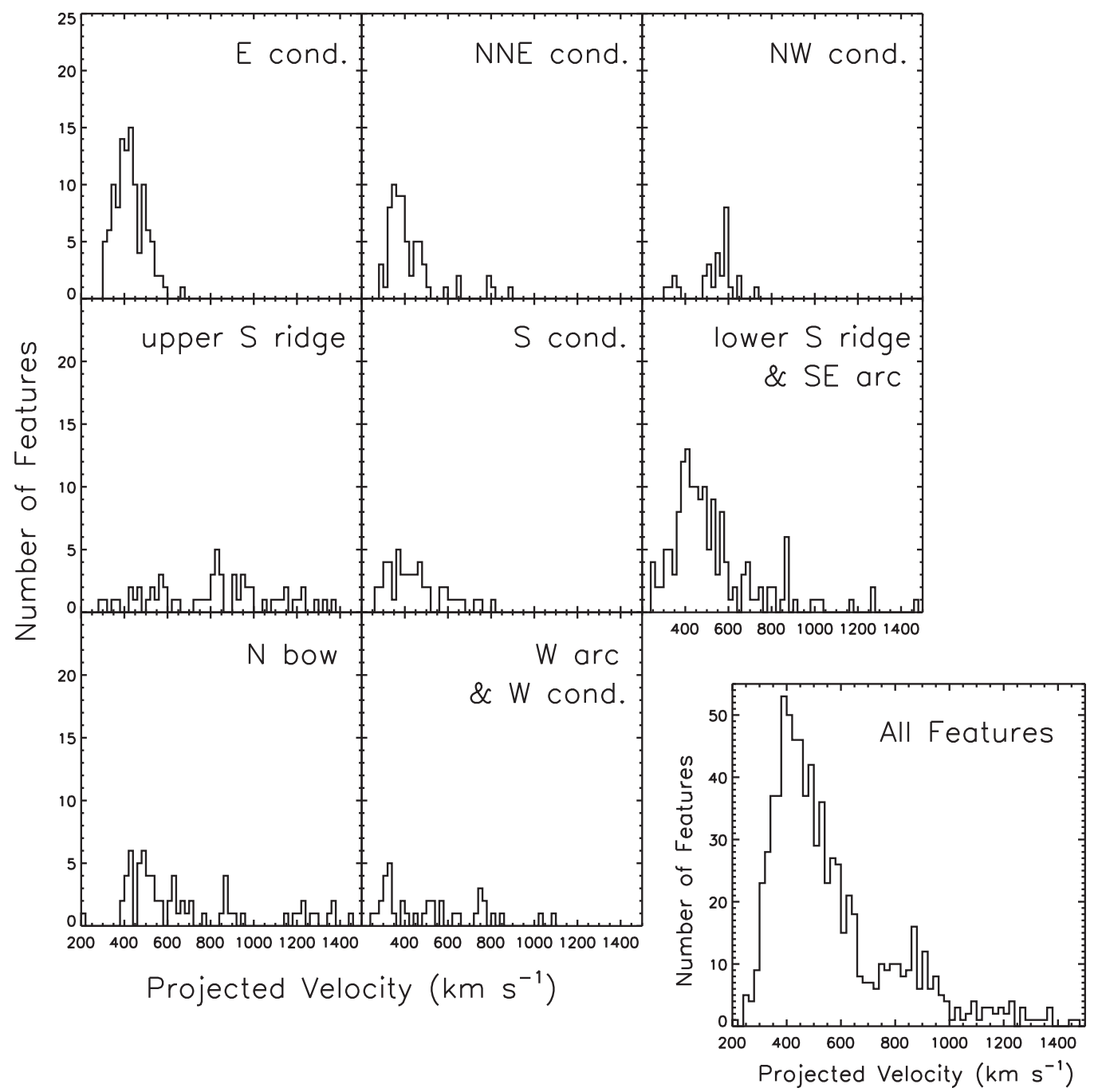

Figure 4. Histograms of the weighted mean proper motions of the outer ejecta, assuming a distance of $2.3 \mathrm{kpc}$. The regions are as shown in Fig. 2; note that some of the regions slightly overlap. The bottom right panel shows the velocity histogram for all 792 measured features.

velocities as constant unless otherwise noted, and use the weighted mean as the final proper motion for each feature.

As a last check on whether the assumption of constant velocities is appropriate, we extrapolate our observed positions of the outer ejecta back to 1950 and compare them to the ground-based photographic plate images of Thackeray (1950). We aligned the Thackeray (1950) images to a copy of our stacked ACS image that had been convolved with a broad PSF to approximately match the resolution of the older images. Fig. 6 shows the predicted 1950 positions, with the observed 2005 positions overplotted for comparison. There is good agreement between our predicted positions and the observed features in 1950. Although one must be conscious of the limitations of photographic plate data, these data are consistent with ballistic motion of $\eta$ Car's ejecta over the past 60 years. From the motion that we measure, however, we cannot rule out significant acceleration or deceleration that may have occurred earlier than 60 years ago.

\subsection{Ages of the outer ejecta}

Under the assumption that the constant velocities observed over the last 60 years apply over the lifetime of the ejecta, estimating ejection dates is trivial. Earlier episodes of acceleration or deceleration would affect the specific ejection dates deduced, but, as we discuss in Section 4.3, are unlikely to affect the overall relationships between groups of ejecta. Ejection date results are summarized in Fig. 7: the magnitude and direction of each knot's proper motion are indicated by the length and orientation of its arrow, and the arrows are colour-coded by apparent ejection date. Red arrows mark the most recently ejected material, i.e. material that was ejected around the time of the Great Eruption, while blue and purple arrows indicate the oldest material. It can be seen in Fig. 7 that all 792 measured features are moving nearly directly away from the central star.

There are several distinct groups of ejecta in Fig. 7:

(i) The $\mathrm{N}$ bow, $\mathrm{S}$ condensation, and $\mathrm{S}$ ridge (which extends nearly completely around the Homunculus) all date back to the early 1800 s, consistent with prior results (Walborn et al. 1978; Ebbets et al. 1993; Morse et al. 2001). As we discuss below, the age of the $\mathrm{S}$ ridge suggests an origin during one of the precursor outbursts to the Great Eruption (seen as spikes in the historical light curve; Smith \& Frew 2011), before the ejection of the Homunculus.

(ii) The E blobs and many of the NNE condensations date back to an ejection event in the thirteenth century. This event was apparently 


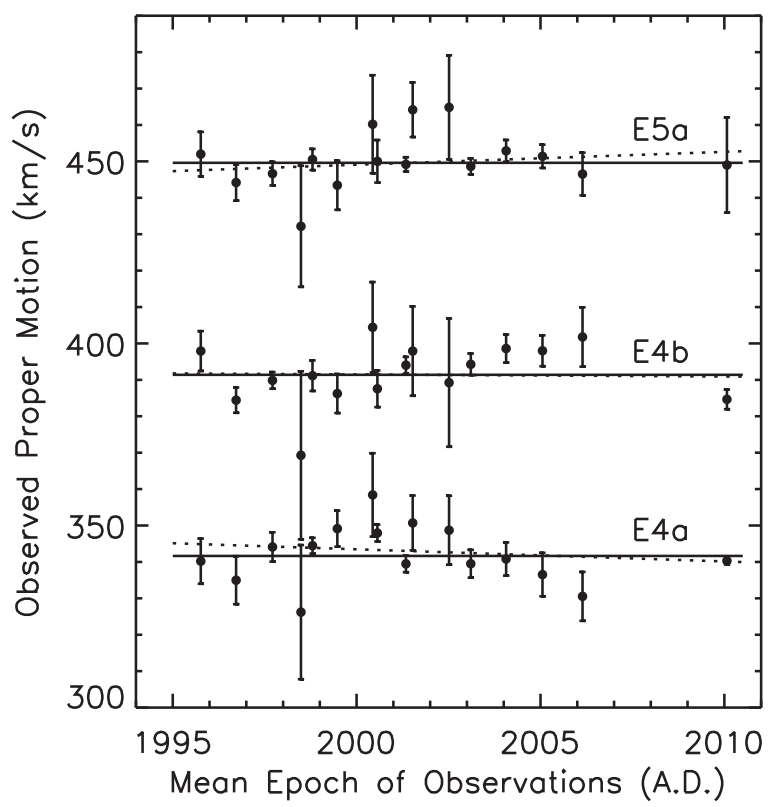

Figure 5. Proper motions measured over all possible baselines for three representative features among the $\mathrm{E}$ condensations. The points around 2010 are from the ACS 2005-2014 baseline, while the other measurements come from the various WFPC2-WFPC2 baselines. The largest uncertainties typically occur over the shortest baselines, e.g. 1997-1999. The solid lines are the weighted means of the measurements, and the dotted lines are leastsquares fits to proper motion versus time for each ejecta feature.

highly asymmetric: there are no ejecta with this age to the south or west. (Note, however, that our HST ACS footprint for this reference frame cuts off in the far southwest. We address this issue further in Section 3.3.) (iii) The SE arc, the W and NW condensations, and part of the $\mathrm{W}$ arc appear to date to an intermediate eruption in the sixteenth century. In the SE and $\mathrm{W}$ arcs, this material is being overtaken by faster-moving material from the Great Eruption. The lower $\mathrm{S}$ ridge is a mix of material of different ages, explaining why Morse et al. (2001) dated this region to half a century before the Great Eruption. One might surmise that the apparently intermediate ages of material in the SE and $\mathrm{W}$ arcs could result from the interaction between material from the 1200 s and material from the 1800s. However, there are no newer ejecta around the NW condensation or the faint ejecta to the far north. As we discuss in Section 4.1, radial velocities also suggest that these ejecta are not connected to the thirteenth-century eruption. They also lie outside the soft Xray shell, which traces strong current interactions between ejecta (see Section 4.2).

Fig. 8 presents these data in an alternative form, plotting the transverse velocity of ejecta features versus their projected distance from $\eta$ Car. Again, there is evidence for at least two eruptions, one in the early 1800 s and another circa 1250 A.D. An intermediate eruption, circa 1550 A.D., is suggested but less obvious. As mentioned above, the sixteenth-century ages could in principle be the result of interaction between ejecta from the Great Eruption and the 1200 s event. However, the intermediate-aged features appear in a fairly clear line in velocity-distance space (i.e. as a Hubble-like law) rather than being smeared between the points from the two other events.

An additional feature of interest in Fig. 8 is that nearly all of the nineteenth-century features appear to have been ejected decades before 1843. Proper motions of the Homunculus date it to the mid1840s (Currie et al. 1996; Smith \& Gehrz 1998; Morse et al. 2001); this is when the light curve of $\eta$ Car reached its peak (Smith \& Frew 2011). There are two possibilities for why the $\mathrm{S}$ ridge and its extended wings appear older: (1) its material experienced a period

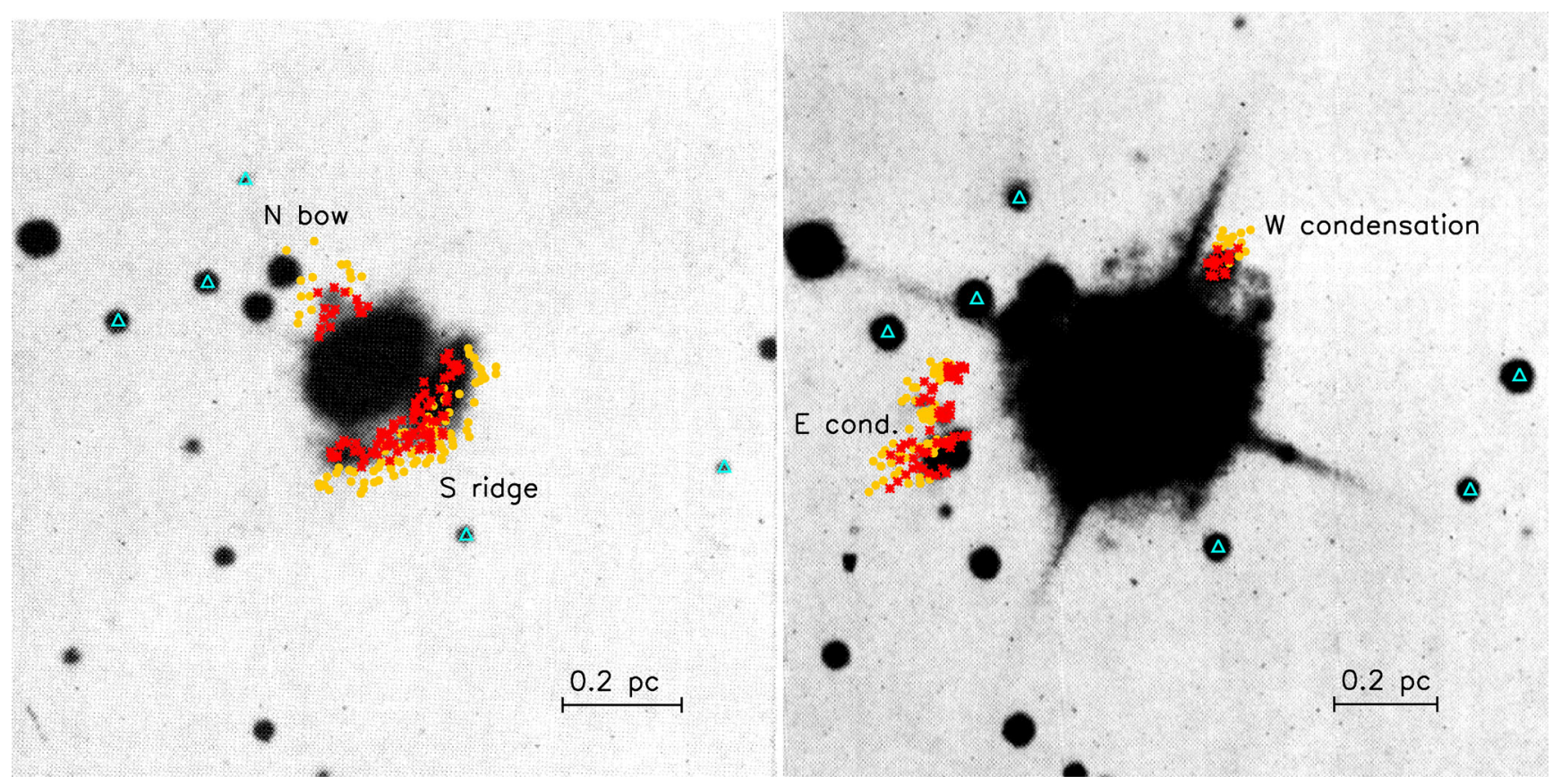

Figure 6. Left: red-sensitive photographic plate image of $\eta$ Car and its surrounding nebulosity from Thackeray (1950). The predicted 1950 positions of the features we observe in the $\mathrm{N}$ bow and $\mathrm{S}$ ridge are plotted as red asterisks, while our observed 2005 positions of the same features are marked with yellow circles. The stars that were used to align this image with our reference frame are indicated with cyan triangles. Right: same as left, using a deeper image from Thackeray (1950) to show the observed current and predicted past positions of the E and $\mathrm{W}$ condensations. 


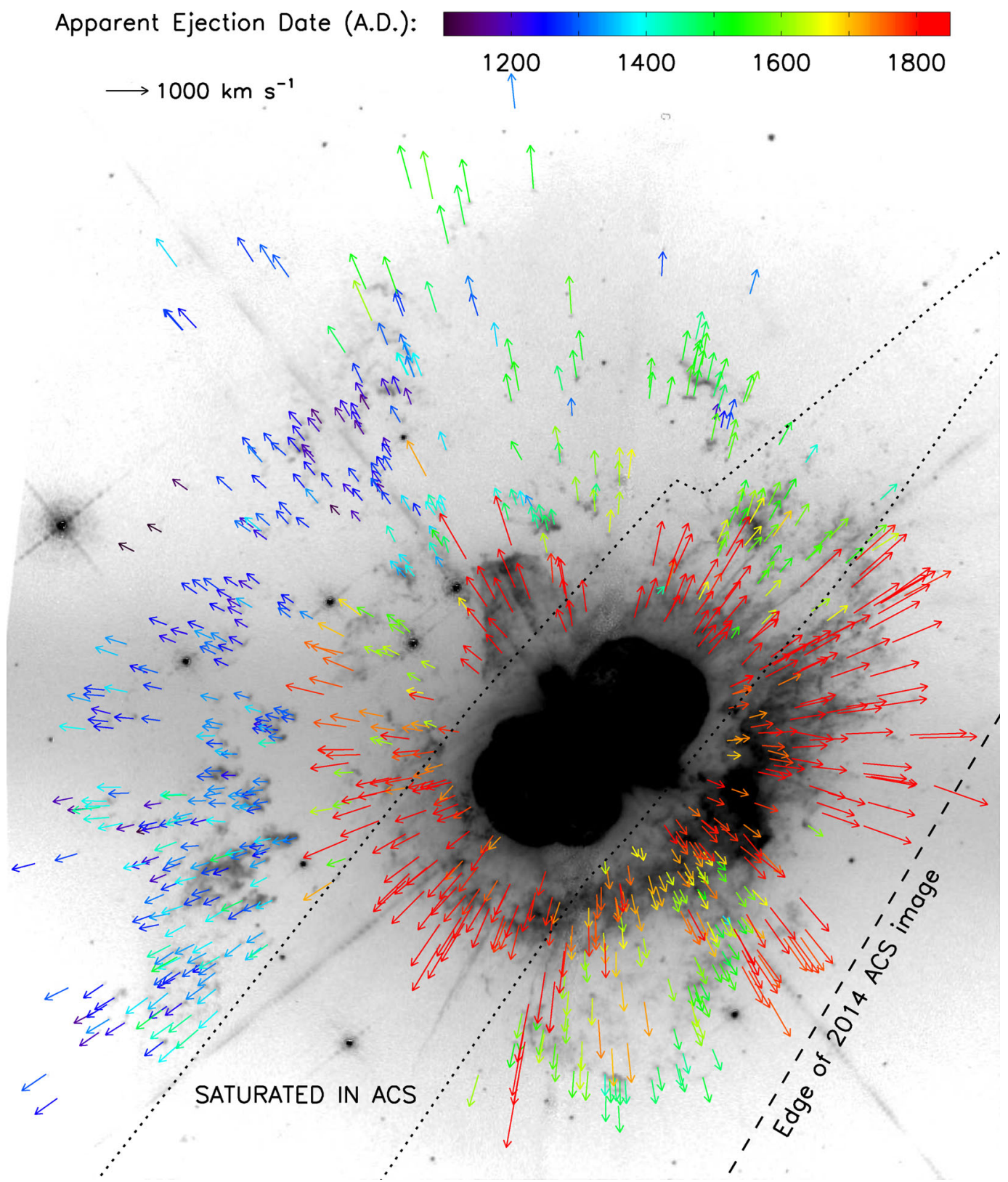

Figure 7. Vectors illustrating the observed proper motions of 792 features in the ejecta of $\eta$ Car. The arrows are colour-coded by the date of ejection from the central star, calculated assuming constant velocity. The region contaminated in the ACS images by bleeding from the highly saturated Homunculus is marked; features in this region were measured in WFPC2 data only. The background image is the same as in Fig. 1.

of deceleration as it interacted with circumstellar material early in its history before continuing to expand ballistically; or (2) it was ejected prior to the formation of the Homunculus. The second option is supported by the photometric record: the Great Eruption was preceded by a brightening in 1838 and a similar but poorly observed event in 1827 (see Smith \& Frew 2011, who found that these events align with the predicted periastron times of $\eta$ Car's binary orbit). The data before 1827 are very sparse (or non-existent) and leave open the possibility of additional prior periastron outbursts. The $\mathrm{S}$ ridge may have been ejected during one or more of these events rather than during the main peak of the Great Eruption. Its strong asymmetry may therefore be related to stellar collisions at periastron (Smith 2011) associated with these early brightenings. The alternative explanation (an early deceleration episode) is plausible for the material at the edges of the bright $\mathrm{S}$ condensation, which appears in Fig. 8 around 16-20 arcsec and 500-700 $\mathrm{km} \mathrm{s}^{-1}$. We could be seeing the result of interaction between the $\mathrm{S}$ condensation (ejected during the Great Eruption) and the slightly older S ridge material.

Finally, Fig. 9 presents histograms of the apparent ejection dates, both overall and for each of the regions in Figs 2 and 4. The differences in age between the various groups of features are clearly evident. The $\mathrm{E}$ and NNE condensations, for instance, are much older than the upper $\mathrm{S}$ ridge and $\mathrm{S}$ condensations. The mix of ages in the $\mathrm{W}$ arc and the lower S ridge is also apparent. As in Fig. 8, the overall histogram of ejection dates shows two obvious distinct events, in the thirteenth and nineteenth centuries, as well as a smaller peak of features ejected in the late $1500 \mathrm{~s}$. 


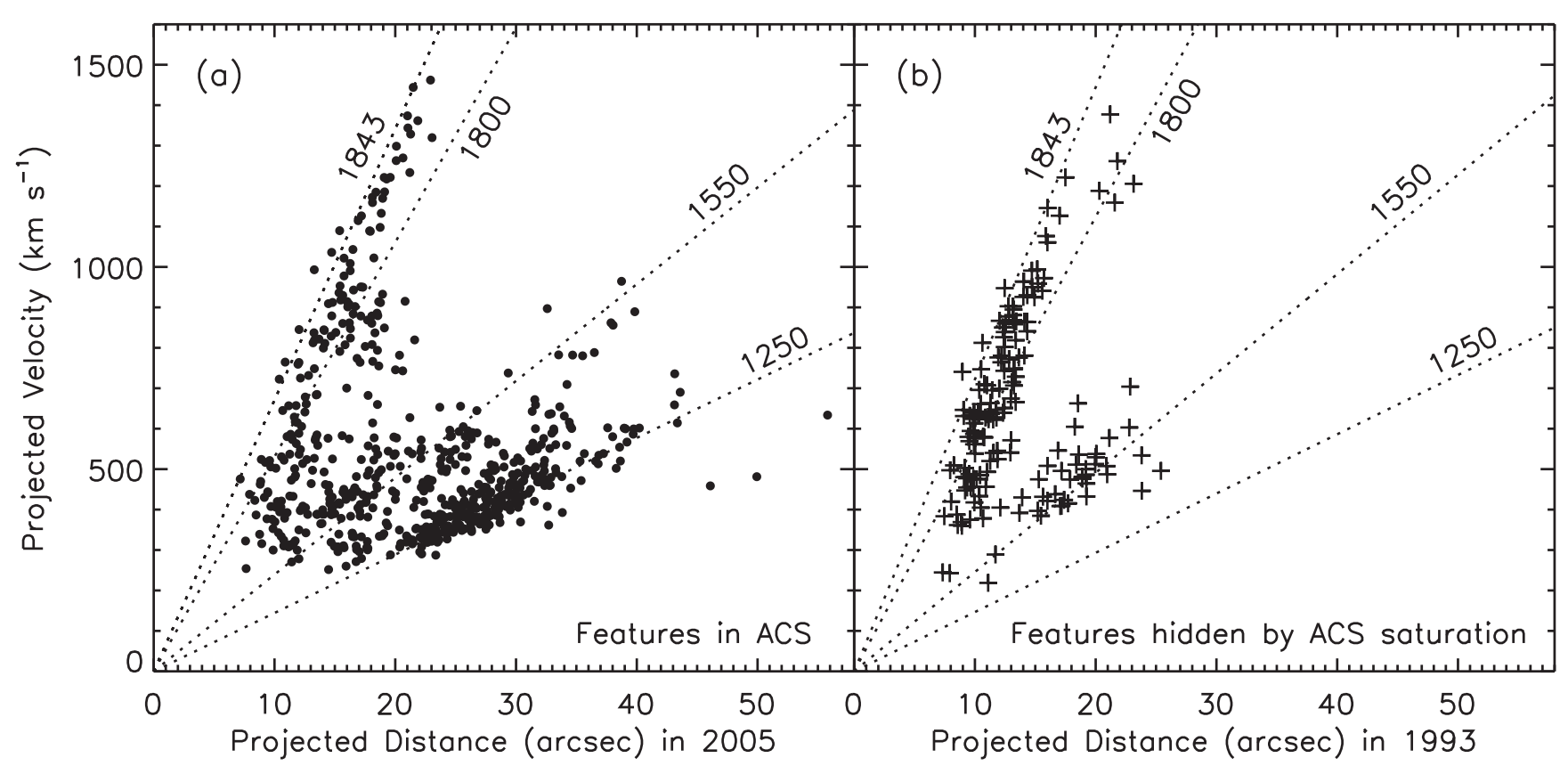

Figure 8. (a) Measured transverse velocity versus projected distance from the central star for all features measured over the ACS $2005-2014$ baseline. The predicted positions of material ejected at the peak of the 1840s Great Eruption is marked, along with tracks for material ejected in 1250 A.D., 1550 A.D., and 1800 A.D., for reference. (b) Same as (a), but for features that were masked by saturation bleeds in the ACS images and were consequently only measured with WFPC2.

Overall, our proper motions of $\eta$ Car's outer ejecta solidly confirm that there was at least one major mass-loss event prior to the Great Eruption, barring a strong deceleration event occurring sometime before 1950 (see Section 4.3). Assuming constant velocities, there is an approximately 600-year interval between the two most distinct eruptions. If the third eruption is included, then these major massloss events have occurred every $\sim 300$ years.

\subsection{Asymmetry and the most distant ejecta}

As mentioned above and as shown in Fig. 7, the reference frame in which we identified and measured ejecta cuts off closer to $\eta$ Car on the southwest than on any other side, complicating our assessment of asymmetry. We searched the adjacent ACS footprints (which together make up a mosaic of the Tr 16 cluster; see Smith et al. 2010) for other possible ejecta. As shown in Fig. 10, we found only four small features moving away from $\eta$ Car, three to the south and one to the north-west. We were able to measure the proper motions of three of those features, although a relative zero-point uncertainty of several $\mathrm{km} \mathrm{s}^{-1}$ exists between footprints because of their minimal overlap. For the same reason, there is also a several-pixel (100s mas) uncertainty in distance from the central source.

The knot to the north-west has a transverse velocity of $\sim 630 \mathrm{~km} \mathrm{~s}^{-1}$, giving it an estimated ejection date of $1045 \pm 15$ A.D., assuming ballistic motion. The two knots that we were able to measure to the far south are travelling at $460-480 \mathrm{~km} \mathrm{~s}^{-1}$ and date to $900 \pm 30$ A.D. The four outer knots could thus come from a smaller, older mass-loss event or events. Given the systemic uncertainties described above, however, we cannot rule out an association with the thirteenth-century event.

Notably, there are no large-scale older ejecta similar to the E condensations on the west side of $\eta$ Car. It would appear that the eruption in the 1200 s was highly asymmetric, perhaps even onesided, sending substantial mass to the east and northeast but little to no mass in other directions. The Great Eruption, despite producing the bipolar Homunculus, was also significantly asymmetric, ejecting more mass into the extended $\mathrm{S}$ ridge than into the distinctly shaped $\mathrm{N}$ bow. The sixteenth-century ejecta show some possible bipolarity, but are not aligned with the axis of the Homunculus.

\section{DISCUSSION}

\subsection{Comparison to radial velocities}

To fill out the third dimension of the outer ejecta's motion, we turn to radial velocity studies in the literature. Weis, Duschl \& Bomans (2001) measured radial velocities of 90 distinct features in the outer ejecta, finding speeds from $100 \mathrm{~km} \mathrm{~s}^{-1}$ (in the SE arc) to $1960 \mathrm{~km} \mathrm{~s}^{-1}$ (in the upper part of the $\mathrm{S}$ ridge). The E condensations, the $\mathrm{N}$ bow, most of the NNE condensations, and the unnamed material on the east side of the Homunculus - including all of the ejecta that date back to the 1200 s - are blueshifted. In contrast, the $\mathrm{S}$ ridge, $\mathrm{S}$ condensation, $\mathrm{SE}$ arc, $\mathrm{W}$ arc, $\mathrm{W}$ condensation, $\mathrm{NW}$ condensation, and some of the material to the far north are redshifted. The Homunculus itself is also aligned in this fashion, with a blueshifted south-east lobe and redshifted north-west lobe, tilted out of the plane of the sky by $48^{\circ}$ (e.g. Davidson et al. 2001; Smith 2006b).

The ejecta from the thirteenth-century eruption are travelling towards $u s$ at an angle of $20-40^{\circ}$ out of the plane of the sky, depending on whether we use the radial velocities of Meaburn, Wolstencroft \& Walsh (1987) and Smith \& Morse (2004) or those of Weis et al. (2001, who present only the highest-magnitude velocities for each feature). The ejecta that appear to date from the 1500 s, however, are travelling away from us, tilted up to $30^{\circ}$ from the plane of the sky. The dividing line between the two proper motion groups on the north side of $\eta$ Car closely follows the dividing line between blue- and redshifted material seen by Weis et al. (2001). With the 


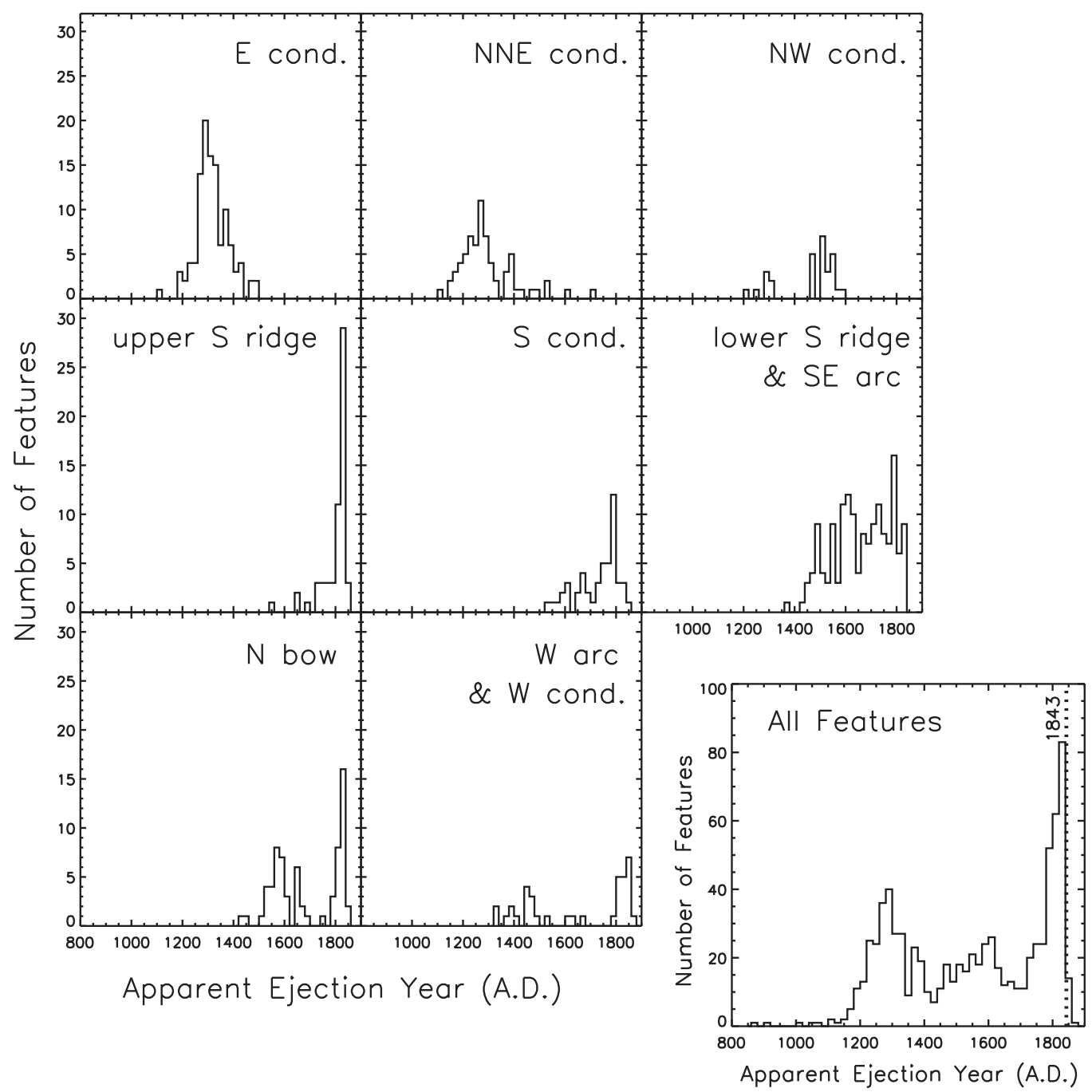

Figure 9. Histograms of the apparent ejection dates of $\eta$ Car's outer ejecta, assuming constant velocity since ejection. The regions are as shown in Fig. 2. The bottom right panel shows the ejection date histogram for all 792 measured features, with the peak of the Great Eruption in 1843 marked by a dotted line.

intermediate-aged material in a completely different part of threedimensional space than the oldest ejecta, it becomes much more likely that the intermediate-aged features are from a distinct eruption rather than the result of past acceleration/deceleration from ejecta interactions.

\subsection{Relationship to X-ray emission and the extremely fast ejecta}

$\eta$ Car is encircled by an elongated partial ring or shell of soft X-ray emission that extends from just south of the $\mathrm{S}$ condensation, over the W arc, and around to the E condensations (Chlebowski et al. 1984; Seward et al. 2001). Fig. 11 shows a Chandra X-ray Observatory image of $\eta$ Car and its surroundings (Seward et al. 2001), with the proper motion velocity vectors for the optical ejecta plotted on top. The X-ray shell has roughly the same axis orientation as the Homunculus, but has a notable gap to the south and southeast.

The spatial association between the soft X-ray shell and the optical features in the outer ejecta has led to a broad consensus that the X-ray emission is the result of shock heating from ejecta interactions (Chlebowski et al. 1984; Corcoran et al. 1998; Seward et al. 2001; Weis et al. 2001; Smith \& Morse 2004). The strongest soft X-ray emission is coincident with the $\mathrm{S}$ condensation, upper $\mathrm{S}$ ridge, and $\mathrm{W}$ arc, where we have measured a mix of ages for the dense optical ejecta. Extremely fast ejecta, with radial speeds of up to $\sim 3000 \mathrm{~km} \mathrm{~s}^{-1}$, have been detected close to the E condensations and coincident with the X-ray shell (Smith \& Morse 2004; Smith 2008). As described in Section 2.2, this very fast material, originating from the Great Eruption in the nineteenth century, is Doppler-shifted out of the narrow-band filters used here and is not detected in our images. It is presumably interacting with or approaching the denser, slower blobs measured in this paper. Additional spectral mapping of the outer ejecta is needed to explore the full distribution of the high-velocity material and its relationship to the soft X-ray emission.

Given the limits on the deceleration that we measured for the knots in the E condensations, we can roughly estimate their relative density compared to the very fast ejecta. Over 21 years of HST observations, the features in the E condensations change velocity by an average of $-0.1 \pm 1.2 \mathrm{~km} \mathrm{~s}^{-1} \mathrm{yr}^{-1}$ (consistent with zero). Assuming that the collision between ejecta is fully inelastic and that momentum is conserved, the observed E condensations are thus 10$10^{4}$ times denser than the impacting very fast ejecta, depending on the time-scale over which the fast ejecta decelerate. 


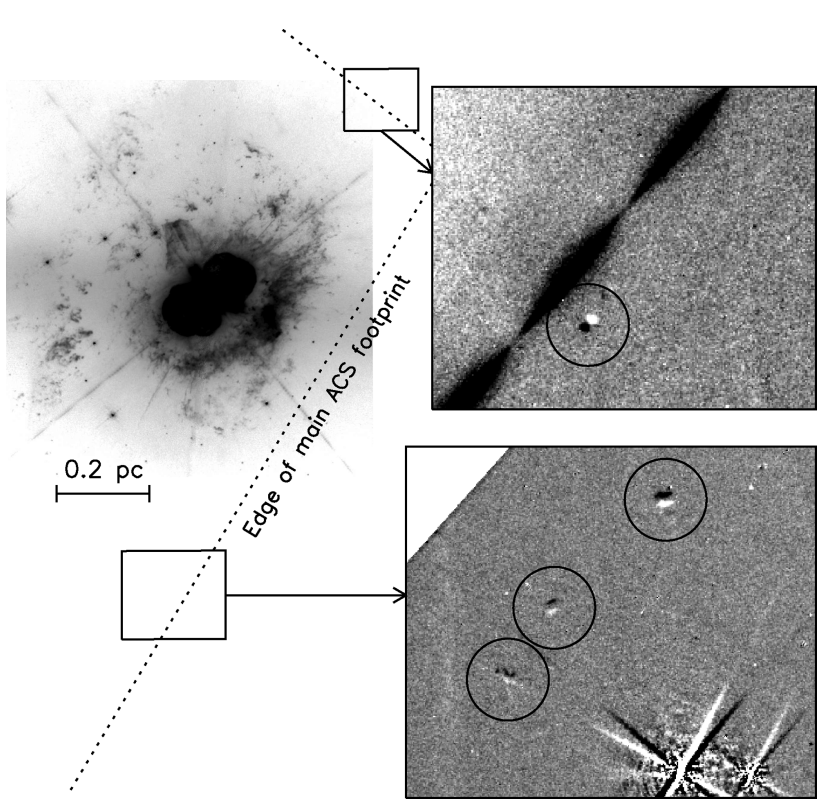

Figure 10. Difference images (ACS 2014 - ACS 2005) showing knots identified in images outside the primary observed footprint. The image from Fig. 1 is shown as a reference; the small boxes indicate where the displayed features are located relative to $\eta$ Car. The four knots showing motion over the 9 -yr baseline are circled.

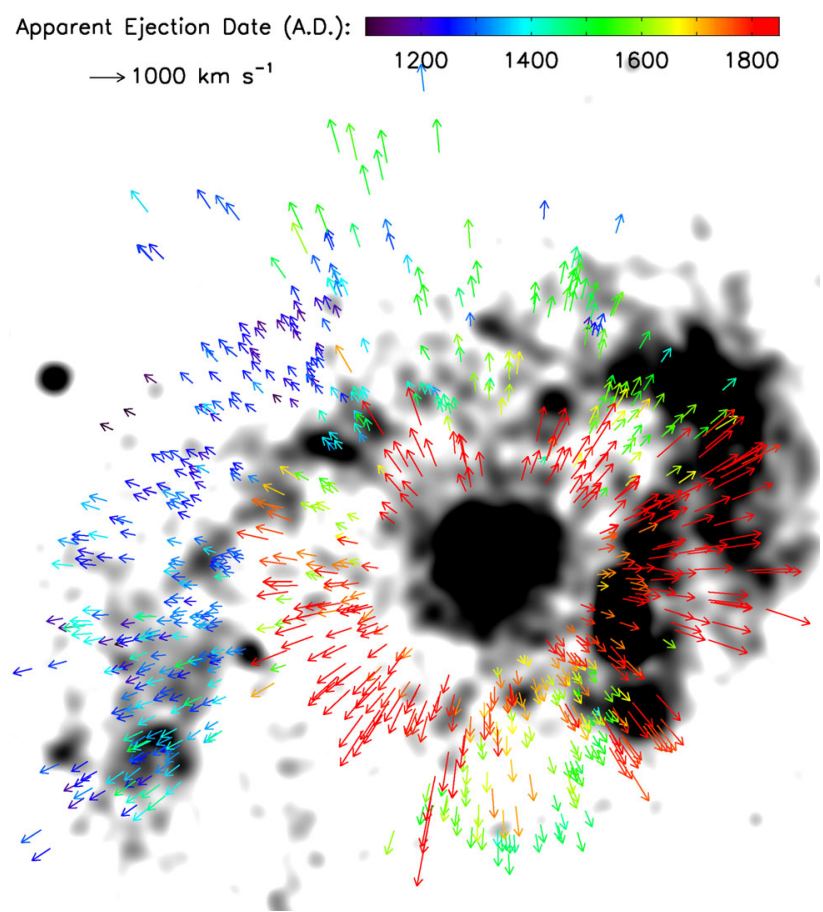

Figure 11. Similar to Fig. 7, with the scaled and colour-coded proper motion vectors overplotted on a soft X-ray Chandra image of $\eta$ Car and its surroundings. This X-ray image (Seward et al. 2001) covers the energy range $0.5-1.5 \mathrm{keV}$ and was adaptively smoothed as in Smith \& Morse (2004).

Returning to Fig. 11, note that there is no spatial correlation between the sixteenth-century ejecta and the soft X-ray emission. The SE arc falls in the X-ray gap, and the northern ejecta lie outside the X-ray shell altogether. If the intermediate ages of these ejecta were the result of interaction between the thirteenth- and nineteenthcentury ejecta, we would expect to see strong X-ray emission from the location of these interactions. We would also expect there to be apparently sixteenth-century ejecta around the E blobs, where very fast ejecta are observed to be hitting the thirteenth-century material. We observe neither of these things, further strengthening the case that there was a distinct eruption in the 1500 s.

\subsection{Alternate ejection histories}

In our analysis, we have found approximate ejection dates by assuming that there was no substantial, large-scale deceleration (or acceleration) of the outer ejecta prior to their first detection in 1950. $\eta$ Car is surrounded by a 'cocoon' of less-nitrogen-rich material, likely from pre-eruption mass loss (Smith \& Morse 2004). Here, we explore the hypothesis that all of the observed outer ejecta were decelerated early in their history as they encountered this material, then tapered to their current coasting velocities as the density of the cocoon diminished with radius. In this case, the ejection dates of all the knots move later and closer together. The true time-scale between eruptions could then be closer to 100-200 years instead of 300 years. However, our main result, the detection of multiple major mass-loss events in $\eta$ Car's history, is unaffected. That is the most straightforward scenario to explain our observations.

The most extreme alternate ejection history - that all of the observed outer ejecta date to the Great Eruption of the 1840s, but suffered different amounts of deceleration with an asymmetric wind is much less likely. It requires a complex pre-existing configuration of mass to decelerate the observed outer ejecta such that they reach their current coasting velocities at their current distances from $\eta$ Car. A spherically symmetric distribution of pre-eruption circumstellar material (e.g. a stellar wind with a $r^{-2}$ density profile) could not have produced the different coasting velocities seen at the same radii from the star. Compare, for example, the NNE and NW condensations: although both are $\sim 25 \operatorname{arcsec}$ from $\eta$ Car, the average proper motion of the NNE knots is $380 \mathrm{~km} \mathrm{~s}^{-1}$ while the NW knots are moving at $550 \mathrm{~km} \mathrm{~s}^{-1}$.

The E condensations provide a particularly firm constraint, as they are already 19-23 arcsec from the central star in the Thackeray (1950) images. To reach that distance by 1950 with an origin in the 1840 s would require a minimum initial projected speed of $\sim 2000 \mathrm{~km} \mathrm{~s}^{-1}$. The measured projected speeds of the E condensations over the past two decades are $350-600 \mathrm{~km} \mathrm{~s}^{-1}$. If these features were ejected in the $1840 \mathrm{~s}$, there is no way for them to have reached the observed separation from $\eta$ Car and yet have their current, relatively low speeds without invoking a dense shell located close to their 1950 positions. One could then posit additional preexisting partial shells that would have decelerated the NW and SE condensations to their present-day coasting velocities. This scenario would still require substantial and episodic prior mass-loss events. It would have much the same consequences for $\eta$ Car's eruptive history, except it would indicate that the star's surface nitrogen enhancement took place recently, between the older shell-producing events and the 1840s Great Eruption.

From an energy standpoint, we know the Great Eruption did produce ejecta with velocities of $1000 \mathrm{~km} \mathrm{~s}^{-1}$ or more; they are seen in spectra of the equatorial plane of the Homunculus and of near or inside the soft X-ray shell (Smith 2008). However, as discussed in Section 4.2 , some of these very fast ejecta are coincident with and appear to be running into the E condensations, likely powering the observed soft X-ray shell. There is no clear mechanism by which two sets of mass should reach the same projected distance over the same time yet have present-day speeds that differ by a factor of 10 . Furthermore, the ejection of all of the dense outer ejecta at 
$1000 \mathrm{~km} \mathrm{~s}^{-1}$ or more would require a dramatic upward revision of the estimated energy budget of the Great Eruption. The discovery of the very fast ejecta roughly doubled the Great Eruption's energy budget (Smith 2008), and that number assumed that the $3000 \mathrm{~km} \mathrm{~s}^{-1}$ material was about a factor of 10 less dense than the slower, named ejecta. (Note that this agrees with our rough estimate of the density ratio in Section 4.2.) The explosive amount of kinetic energy required to eject several solar masses of outer ejecta at these speeds approaches that of a core-collapse supernova.

\subsection{Implications for the models of Eta Car}

The proper motions of the outer ejecta thus confirm that $\eta$ Car has undergone at least two and probably three major mass-loss events over the last millennium, including the nineteenth-century Great Eruption. These results raise a number of interesting questions that are as yet unanswered by existing theories: what mechanism drives these repeating eruptions? why do they repeat on a several-hundredyear time-scale, much longer than the 5.5-year binary orbit? why was the thirteenth-century eruption so one-sided? why does the Homunculus have a clear bipolar symmetry that past eruptions lack? Models of $\eta$ Car's behaviour must not treat the Great Eruption in isolation, but should aim to account for all of the observed characteristics of the prior eruptions.

In single-star models (i.e. those in which the companion star plays no significant role), $\eta$ Car's eruptions are considered to be continuum-driven super-Eddington wind events, where the extra luminosity arises from as-yet-unidentified mechanisms (Davidson \& Humphreys 1997; Owocki et al. 2004). However, it is difficult for a single-star model to explain the observed asymmetry in the outer ejecta and the changes in mass-loss symmetry with time. What could cause a single star to produce the highly one-sided mass loss of the thirteenth-century eruption, then eject the axisymmetric Homunculus several centuries later?

Current models involving a binary or higher-order multiple system may get us closer, but also have difficulty explaining the observed historical mass loss. The time between major eruptive events is much longer than the orbital period of the current binary (5.54 yr; Damineli 1996). If the eruptions are influenced by periastron interactions (Cassinelli 1999; Soker 2007; Kashi \& Soker 2010; Smith 2011), these interactions must be suppressed for long periods of time. Models that invoke a one-time catastrophic event such as a merger (Gallagher 1989; Iben 1999; Morris \& Podsiadlowski 2009; Podsiadlowski 2010; Portegies Zwart \& van den Heuvel 2016) or a dynamical exchange (Livio \& Pringle 1998) require additional unexplained complexities to account for these repetitive yet discrete events separated by centuries.

The lack of older, more distant outer ejecta rules out additional major mass-loss events prior to the thirteenth century. Bohigas et al. (2000) claimed to detect a much older $\left(10^{4} \mathrm{yr}\right)$ bipolar shell, but this was interpreted by Smith, Morse \& Bally (2005) as $\eta$ Car's astropause, modified by the wind of a nearby Of-type star, as it lacks the nitrogen-rich chemistry or clumpy structure of the other outer ejecta. What, then, caused $\eta$ Car's eruptive behaviour to start in the 1200s? The apparently sudden initiation of mass ejections is probably an important clue, and is another key constraint for models.

An added complication in the story of $\eta$ Car is the 1890s Lesser Eruption, in which a much smaller amount of mass $\left(0.1 \mathrm{M}_{\odot}\right.$; Ishibashi et al. 2003; Smith 2005) was ejected with the same geometry as the Homunculus. Secondary eruptions of this sort, occurring several decades after a major outburst, have been observed in $\mathrm{P}$ Cygni and some of the other known eruptive LBVs (Humphreys et al. 1999). Our data are not sensitive to similar small eruptions in the decades after the larger thirteenth- and sixteenth-century events.

\section{CONCLUSIONS}

We have aligned eight epochs of HST imaging (both WFPC2 and ACS) of $\eta$ Car's outer ejecta to the same distortion-corrected reference frame and measured the proper motions of 792 ejecta features, many for the first time. We achieve unprecedented time coverage, with each feature measured in up to 16 baselines over 21 years, as well as unprecedented velocity precision (few $\mathrm{km} \mathrm{s}^{-1}$ ) and spatial resolution for these features.

All 792 features measured in $\eta$ Car's outer ejecta have transverse velocities pointing nearly directly away from the star. The majority have proper motions of $300-600 \mathrm{~km} \mathrm{~s}^{-1}$, although some are as fast as $1500 \mathrm{~km} \mathrm{~s}^{-1}$. The fastest-moving material is found in the large feature known as the $\mathrm{S}$ ridge and in the broadly jet-shaped $\mathrm{N}$ bow. Both date back to $\eta$ Car's Great Eruption in the 1840s or to a few decades prior.

Over the 21 years of data, we see no evidence for large-scale acceleration or deceleration of any of the outer ejecta: 94 per cent of the knots are consistent with moving at constant velocity over that time. Comparison to images from 1949-1950 support ballistic motion over a longer time period. Under the assumption of constant velocity, we find that material in and around the E and NNE condensations was ejected in the mid-1200s A.D., give or take 50 100 years. With the exception of three small knots to the far south of $\eta$ Car and one to the north-west, the ejecta dating to the mid-1200s are all found to one side of the central star and are blueshifted.

We also see evidence of a third, intermediate eruption that occurred in the sixteenth century. Ejecta dating to the mid-1500s are found in the SE arc, the W condensation, and in and around the NW condensation. From proper motions alone, we cannot rule out that this intermediate date peak is the result of newer ejecta from the Great Eruption hitting the older material from the 1200s. However, the radial velocities of these features place them in a different part of three-dimensional space from the thirteenth-century ejecta. The lack of X-ray emission over the SE arc and the features to the far north also indicates a lack of strong interaction between ejecta at those spots.

In summary, we have shown with distance-independent measurements that $\eta$ Car erupted at least once, likely twice, before its Great Eruption in the 1800s. Models for this still-enigmatic star must therefore explain the recurrence of these major mass-loss events, along with their several-hundred-year time-scale and their various asymmetries.

\section{ACKNOWLEDGEMENTS}

The authors would like to thank Jay Anderson for providing us with his suite of PSF-fitting and image alignment software, and for his valuable instruction, guidance, and technical support. We also thank the anonymous referee for constructive comments that improved the paper. Support for this work was provided by NASA grant GO-13390 from the Space Telescope Science Institute, which is operated by the Association of Universities for Research in Astronomy, Inc. under NASA contract NAS 5-26555. This work is based on observations made with the NASA/ESA Hubble Space Telescope, obtained from the Data Archive at the Space Telescope Science Institute. 


\section{REFERENCES}

Anderson J., 2006, in Koekemoer A. M., Goudfrooij P., Dressel L. L., eds, The 2005 HST Calibration Workshop: Hubble After the Transition to Two-Gyro Mode. Space Telescope Science Institute, Baltimore, MD, p. 11

Anderson J., King I. R., 1999, PASP, 111, 1095

Anderson J., King I. R., 2000, PASP, 112, 1360

Anderson J., King I. R., 2006, Technical Report, PSFs, Photometry, and Astronomy for the ACS/WFC. Space Telescope Science Institute, Baltimore, MD, p. 1

Anderson J., van der Marel R. P., 2010, ApJ, 710, 1032

Anderson J. et al., 2008a, AJ, 135, 2055

Anderson J. et al., 2008b, AJ, 135, 2114

Bohigas J., Tapia M., Ruiz M. T., Roth M., 2000, MNRAS, 312, 295

Cassinelli J. P., 1999, in Morse J. A., Humphreys R. M., Damineli A., eds, Astronomical Society of the Pacific Conference Series Vol. 179, Eta Carinae at The Millennium. Astron. Soc. Pac., San Francisco, p. 358

Chlebowski T., Seward F. D., Swank J., Szymkowiak A., 1984, ApJ, 281, 665

Corcoran M. F. et al., 1998, ApJ, 494, 381

Corcoran M. F., Ishibashi K., Swank J. H., Petre R., 2001, ApJ, 547, 1034

Currie D. G. et al., 1996, AJ, 112, 1115

Currie D. G., Dorland B. N., Kaufer A., 2002, A\&A, 389, L65

Damineli A., 1996, ApJ, 460, L49

Damineli A., Conti P. S., Lopes D. F., 1997, New Astron., 2, 107

Damineli A., Kaufer A., Wolf B., Stahl O., Lopes D. F., de Araújo F. X., 2000, ApJ, 528, L101

Davidson K., 1971, MNRAS, 154, 415

Davidson K., Humphreys R. M., 1997, ARA\&A, 35, 1

Davidson K., Dufour R. J., Walborn N. R., Gull T. R., 1986, ApJ, 305, 867

Davidson K., Smith N., Gull T. R., Ishibashi K., Hillier D. J., 2001, AJ, 121, 1569

de Jager C., 1984, A\&A, 138, 246

Ebbets D., Malumuth E., Davidson K., White R., Walborn N., 1993, in Cassinelli J. P., Churchwell E. B., eds, Astronomical Society of the Pacific Conference Series Vol. 35, Massive Stars: Their Lives in the Interstellar Medium. Astron. Soc. Pac., San Francisco, p. 263

Frew D. J., 2004, J. Astron. Data, 10, 6

Gallagher J. S., 1989, in Davidson K., Moffat A. F. J., Lamers H. J. G. L. M., eds, Astrophysics and Space Science Library, Vol. 157. IAU Colloq. 113: Physics of Luminous Blue Variables. Kluwer, Dordrecht, p. 185

Glatzel W., 1994, MNRAS, 271, 66

Glatzel W., Kiriakidis M., 1993, MNRAS, 263, 375

Gomez H. L., Vlahakis C., Stretch C. M., Dunne L., Eales S. A., Beelen A., Gomez E. L., Edmunds M. G., 2010, MNRAS, 401, L48

Hartigan P., Morse J. A., Reipurth B., Heathcote S., Bally J., 2001, ApJ, 559, L157

Humphreys R. M., Davidson K., 1994, PASP, 106, 1025

Humphreys R. M., Davidson K., Smith N., 1999, PASP, 111, 1124

Iben I., Jr, 1999, in Morse J. A., Humphreys R. M., Damineli A., eds, Astronomical Society of the Pacific Conference Series Vol. 179, Eta Carinae at The Millennium. Astron. Soc. Pac., San Francisco, p. 367

Innes R. T. A., 1903, Ann. Cape Obser., 9, 75

Ishibashi K. et al., 2003, AJ, 125, 3222

Kashi A., Soker N., 2010, ApJ, 723, 602

Lamers H. J. G. L. M., Fitzpatrick E. L., 1988, ApJ, 324, 279

Livio M., Pringle J. E., 1998, MNRAS, 295, L59

Maeder A., 1983, A\&A, 120, 113

Meaburn J., Wolstencroft R. D., Walsh J. R., 1987, A\&A, 181, 333

Meaburn J., Boumis P., Walsh J. R., Steffen W., Holloway A. J., Williams R. J. R., Bryce M., 1996, MNRAS, 282, 1313

Morris T., Podsiadlowski P., 2009, MNRAS, 399, 515

Morse J. A., 1999, in Morse J. A., Humphreys R. M., Damineli A., eds, Astronomical Society of the Pacific Conference Series Vol. 179, Eta Carinae at The Millennium. Astron. Soc. Pac., San Francisco, p. 13
Morse J. A., Davidson K., Bally J., Ebbets D., Balick B., Frank A., 1998, AJ, 116, 2443

Morse J. A., Kellogg J. R., Bally J., Davidson K., Balick B., Ebbets D., 2001, ApJ, 548, L207

Owocki S. P., Gayley K. G., Shaviv N. J., 2004, ApJ, 616, 525

Podsiadlowski P., 2010, New Astron. Rev., 54, 39

Portegies Zwart S. F., van den Heuvel E. P. J., 2016, MNRAS, 456, 3401

Reiter M., Smith N., 2014, MNRAS, 445, 3939

Reiter M., Smith N., Kiminki M. M., Bally J., Anderson J., 2015a, MNRAS, 448, 3429

Reiter M., Smith N., Kiminki M. M., Bally J., 2015b, MNRAS, 450, 564

Seward F. D., Butt Y. M., Karovska M., Prestwich A., Schlegel E. M., Corcoran M., 2001, ApJ, 553, 832

Shaviv N. J., 2000, ApJ, 532, L137

Smith N., 2005, MNRAS, 357, 1330

Smith N., 2006a, MNRAS, 367, 763

Smith N., 2006b, ApJ, 644, 1151

Smith N., 2008, Nature, 455, 201

Smith N., 2011, MNRAS, 415, 2020

Smith N., Frew D. J., 2011, MNRAS, 415, 2009

Smith N., Gehrz R. D., 1998, AJ, 116, 823

Smith N., Hartigan P., 2006, ApJ, 638, 1045

Smith N., Morse J. A., 2004, ApJ, 605, 854

Smith N., Tombleson R., 2015, MNRAS, 447, 598

Smith N., Gehrz R. D., Hinz P. M., Hoffmann W. F., Hora J. L., Mamajek E. E., Meyer M. R., 2003, AJ, 125, 1458

Smith N., Morse J. A., Bally J., 2005, AJ, 130, 1778

Smith N., Bally J., Walborn N. R., 2010, MNRAS, 405, 1153

Sohn S. T., Anderson J., van der Marel R. P., 2012, ApJ, 753, 7

Soker N., 2007, ApJ, 661, 490

Stothers R. B., Chin C.-W., 1993, ApJ, 408, L85

Thackeray A. D., 1950, MNRAS, 110, 524

van Genderen A. M., 2001, A\&A, 366, 508

Walborn N. R., 1976, ApJ, 204, L17

Walborn N. R., Blanco B. M., 1988, PASP, 100, 797

Walborn N. R., Blanco B. M., Thackeray A. D., 1978, ApJ, 219, 498

Weis K., 2012, in Davidson K., Humphreys R. M., eds, Astrophysics and Space Science Library, Vol. 384. Astrophysics and Space Science Library. p. 171

Weis K., Duschl W. J., Bomans D. J., 2001, A\&A, 367, 566

Whitelock P. A., Feast M. W., Marang F., Breedt E., 2004, MNRAS, 352, 447

\section{SUPPORTING INFORMATION}

Additional Supporting Information may be found in the online version of this article:

\section{kiminki2016_supp.tar.gz}

(http://www.mnras.oxfordjournals.org/lookup/suppl/doi:10.1093/ mnras/stw2019/-/DC1).

Please note: Oxford University Press is not responsible for the content or functionality of any supporting materials supplied by the authors. Any queries (other than missing material) should be directed to the corresponding author for the article.

This paper has been typeset from a $\mathrm{T}_{\mathrm{E}} \mathrm{X} / \mathrm{L} \mathrm{T} \mathrm{E} \mathrm{X}$ file prepared by the author. 